\title{
Tipo e modo respiratório de futuros profissionais da voz
}

\author{
Carla Aparecida Cielo* Carla Franco Hoffmann** Talita Scherer ${ }^{* *}$ \\ Mara Keli Christmann ${ }^{\star \star \star \star}$
}

Resumo: Objetivo - verificar o tipo e o modo respiratório de futuros profissionais da voz. Métodos - 62 sujeitos, entre 18 e 35 anos (12 do sexo masculino e 50 do sexo femino), avaliados através da escala vocal perceptivoauditiva RASATI, com cálculo de confiabilidade; avaliação do tipo e modo respiratório durante a fala e em repouso. Análise estatística com os testes binomial e qui-quadrado. Resultados $82,3 \%$ dos participantes eram do sexo feminino, não houve diferença significativa comparando-se os tipos e modos respiratórios em repouso e durante a fala em relação aos sexos. A maioria $(80,6 \%)$ apresentou tipo respiratório misto em repouso e durante a fala, e modo respiratório nasal $(90,3 \%)$ em repouso e oronasal $(93,5 \%)$ durante a fala. Conclusão - A maioria dos sujeitos apresentou modo respiratório adequado, porém, tipo respiratório misto. Este é inadequado à grande demanda vocal, sugerindo que seja um aspecto a ser trabalhado com futuros profissionais da voz para evitar possíveis distúrbios vocais.

Descritores: Voz; Respiração; Laringe; Saúde do Trabalhador.

\section{Type and mode respiratory of future voice professionals}

Abstract: Purpose - to check type and mode respiratory of future voice professionals. Methods - 62 subjects, between 18 and 35 years (12 men, mean 24,25 years and 50 women, mean 21,42 years), were evaluated by the RASATI scale, with calculation of reliability, selected according to the criteria inclusion and exclusion were assessed with regard to breath type and breath mode during speech and at rest. We applied binomial tests and chi-square test to check the statistical significance of the findings. Results $-82,3 \%$ of subjects studied was comprised of women, no significant difference when comparingthe breath type and breath mode at rest and during speech studied in relation to the sexes. Most hadsignificant respiratory mixed type $(80,6 \%)$ at rest and during speech, and nasal breath mode $(90,3 \%)$ atrest and oronasal breath mode during speech $(93,5 \%)$. Conclusion - Most subjects had adequatebreath mode, however, respiratory mixed type. This is inappropriate for large vocal demand, suggestingthat this is an aspect to be worked with future voice professional to avoid possible vocal disorders.

Descriptors: Voice; Respiration; Larynx; Occupational Health.

*Doutora em Linguística Aplicada pela Universidade Católica do Rio Grande do Sul (PUCRS), Porto Alegre, RS, Brasil **Mestranda em Distúrbios da Comunicação Humana na Universidade Federal de Santa Maria (UFSM), Santa Maria, RS, Brasil

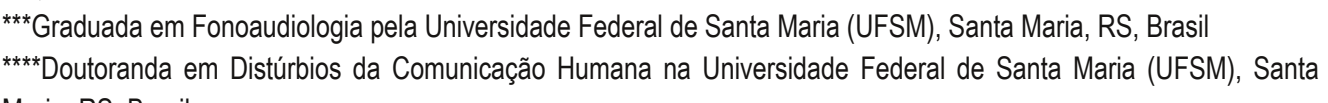
Maria, RS, Brasil

Rev. Saúde (Santa Maria), v.39, n.1, p. 121

130, Jan./Jul. 2013.

Hoffmann, C. et al.

ISSN $2236-5843$ 


\section{Introdução}

Os distúrbios vocais são caracterizados por desvios que comprometem a inteligibilidade e a efetividade da comunicação oral. Podem influenciar diretamente a vida profissional e social do indivíduo, resultando da interação entre fatores hereditários, comportamentais e de estilo de vida. Os fatores comportamentais estão frequentemente ligados à atividade ocupacional, o que pode desencadear até mesmo lesões nas pregas vocais ${ }^{1-4}$.

Como exemplo destes fatores, há o comprometimento da função aérea, base da produção vocal, que poderá exercer efeito deletério sobre as características de loudness, pitch e qualidade da voz ${ }^{1,5-7}$. Outro aspecto importante relacionado à respiração é o modo respiratório, uma vez que a respiração oral ou oronasal é responsável, muitas vezes, pelo surgimento ou agravamento dos distúrbios vocais $2,7-10$.

Estudos com futuros profissionais da voz têm sua importância devido ao fato de possibilitarem a identificação precoce de aspectos alterados relacionados à produção vocal. Dessa forma, torna-se possível a realização de ações de caráter preventivo, tratando os fatores de risco que poderão ser agentes causadores de distúrbios vocais nesta população $0^{3,4,11-15}$. O futuro profissional da voz deverá estar preparado para o aumento da demanda vocal que sua profissão exigirá, sem apresentar problemas com sua resistência vocal que o levem a distúrbios vocais, às lesões de pregas vocais e ou ao afastamento do trabalho devido à voz.

$\mathrm{Na}$ dinâmica respiratória, consideram-se quatro padrões de tipo respiratório: superior, inferior, misto e costodiafragmaticoabdominal (CDA), sendo o último preconizado como 0 ideal durante 0 uso profissional da voz 2,8,10,11,16. Dois estudos com diferentes categorias de profissionais da voz encontraram predominância de tipo respiratório misto e modo nasal nos sujeitos ${ }^{10,11}$, porém no meio científico há poucos estudos que tratam dos padrões respiratórios em profissionais da voz.

Considerando a escassez e a importância de estudos com tal população, principalmente sobre os aspectos respiratórios, este estudo teve por objetivo verificar o tipo e o modo respiratório de futuros profissionais da voz.

\section{Metodologia}

\section{Caracterização da pesquisa, aspectos éticos e seleção dos participantes}

Estudo de observação transversal analítico quantitativo, previamente aprovado pelo Comitê de Ética em Pesquisa da instituição de origem (016945/2010-76). A população-alvo recebeu esclarecimentos sobre a pesquisa e assinou o Termo de Consentimento Livre e Esclarecido (TCLE) (CONEP/1996).

A população estudada consistiu em sujeitos que procuraram a clínica-escola de fonoaudiologia, interessados em realizar avaliação da voz. O período considerado para a

Saúde (Santa Maria), v.39, n.1, p. 121-130, 2013. captação dos sujeitos foi de julho de 2009 a julho de 2010. A amostra deste estudo foi constituída considerando-se os critérios a seguir.

Critérios de inclusão: aderir ao TCLE; ser estudante de curso que exigirá 0 uso profissional da voz futuramente; ter idade entre 18 e 40 anos, evitando-se a influência das ISSN 2236- alterações laríngeas decorrentes do período da muda vocal e do envelhecimento 2,15,17-19; 
apresentar limiares auditivos normais ${ }^{15,17-23}$; apresentar o grau médio de zero a 0,9 nos aspectos avaliados pela escala vocal perceptivoauditiva RASATI ${ }^{15,23}$.

Critérios de exclusão: histórico de qualquer doença neurológica, psiquiátrica, endocrinológica, gástrica, respiratória ou, ainda, alterações do sistema estomatognático que pudessem influenciar o desempenho ou o entendimento das ordens durante as avaliações 2,15,17-19,24; ausência de gripe, alergias respiratórias, alterações hormonais decorrentes de gravidez, período menstrual e pré-menstrual, no dia das avaliações fonoaudiológicas, porque essas situações podem causar alterações temporárias nas pregas vocais, 15,17-20,24,25; ter realizado tratamento fonoaudiológico e/ou otorrinolaringológico prévios, ou cantar em coros, para descartar possível condicionamento vocal e respiratório através de treinamento com técnicas vocais ${ }^{15,18,19}$.

Para a aplicação dos critérios descritos, realizou-se entrevista; triagem audiométrica, por meio da varredura de tons puros, por via aérea nas frequências de 500,1000, 2000 e 4000Hz a $25 \mathrm{~dB}$, em cabine acusticamente tratada (audiômetro Fonix, modelo FA -12, tipo I) ${ }^{15,23}$; avaliação do sistema estomatognático (identificação do aspecto, tensão, postura e mobilidade das estruturas e suas funções); avaliação vocal perceptivoauditiva através da escala RASATI26, para determinação da normalidade das vozes ${ }^{15,25}$.

A escala RASATI avalia os seguintes aspectos: rouquidão, aspereza, soprosidade, astenia, tensão e instabilidade, estabelecendo gradação de "zero" para ausência de alteração, "um" para alteração discreta ou em caso de dúvida se a alteração está presente, "dois" para alteração moderada, e "três" para alteração intensa. É considerada eficaz na identificação perceptivoauditiva de distúrbios vocais de fonte glótica'1,15,20,27,28.

Três juízas, com experiência na área de voz, realizaram individualmente a avaliação perceptivoauditiva sobre o tempo máximo de fonação (TMF) da vogal /a/, sem treinamento prévio. As amostras de voz dos participantes foram randomizadas e apresentadas, sem identificação, duas vezes a cada juíza. As juízas não tiveram conhecimento dos objetivos ou da população-alvo do estudo2,20,25,27. Para o TMF da vogal [a], foi empregado o gravador digital profissional Zoom H4n com microfone estéreo e unidirecional $(96 \mathrm{KHz}, 16 \mathrm{bits})$ a quatro centímetros e a $90^{\circ}$ da boca ${ }^{15,18,23} \mathrm{em}$ sala com ruído ambiental inferior a 50dB NPS (medidor de nível de pressão sonora digital Instrutherm Dec-480)2,15,18,19.

A RASATI determinou a classificação das vozes em normais ou disfônicas, definindo 0 critério de inclusão de vozes normais, uma vez que não foi possível a realização da avaliação otorrinolaringológica em todos os participantes ${ }^{22}$. 0 critério de normalidade estabelecido nesta pesquisa foi o grau médio de até 0,9 nos aspectos avaliados pela escala ${ }^{15,21,22,27}$.

Foram avaliados 86 futuros profissionais da voz, sendo que cinco foram excluídos por terem idade inferior a 18 anos; um por perda auditiva e 18 por apresentarem grau médio superior a 0,9 nos aspectos avaliados pela RASATI. Ao final, a amostra foi composta por 62 participantes: 12 homens, com idades entre 20 e 35 anos (média 24,25) e 50 mulheres, com idades entre 18 e 29 anos (média 21,42).

Coleta de dados

O tipo respiratório foi classificado como superior, inferior, misto ou costodiafragmaticoabdominal (CDA), por meio da observação do sujeito em repouso e durante a fala semiespontânea na entrevista. 0 participante não sabia que estava sendo avaliado, para que não alterasse sua dinâmica respiratória natural $2,8,10,11,13,16$.

Rev. Saúde (Santa Maria), v.39, n.1, p. 121 .

130, Jan./Jul. 2013 Hoffmann, C. et al. 
A avaliação do modo respiratório foi realizada com os participantes em repouso, observando-se a posição dos lábios. Quando os lábios apresentavam-se fechados, a respiração foi classificada como nasal, quando abertos, classificada como oral, e na alternância dessas posições, classificada como oronasal. Os mesmos aspectos foram observados durante a fala 9,29.

Os participantes não foram avaliados exercendo sua atividade profissional, pois estavam em processo de formação como futuros profissionais da voz.

\section{Análise dos dados}

Foi aplicado cálculo do coeficiente Kappa, para verificar a concordância inter e intraavaliador. Consideraram-se os valores entre 0,8 e 1,0 como concordância quase perfeita; entre 0,6 e 0,8, boa; entre 0,4 e 0,6, moderada; entre 0,2 e 0,4, regular; entre zero e 0,2, discreta; entre -1 e zero pobre 15. Realizou-se o cálculo para cada um dos parâmetros da RASATI, obtendo-se o coeficiente médio da concordância inter e intra-avaliador. Para a juíza um (J1), o coeficiente Kappa médio foi de 0,7; para a juíza dois (J2), foi de 0,68 e para a juíza três (J3), foi de 0,71. J1e J2 apresentaram concordância interavaliador de 0,66; J1 e J3 de 0,68 e J2 e J3 de 0,62. Todos os resultados inter e intra-avaliador foram considerados bons.

Após verificar a normalidade das variáveis (teste Lilliefords), optou-se pelo teste binomial para verificar a diferença entre as proporções de cada classificação e pelo teste de associação qui-quadrado para análise entre a variável independente, sexo, e a variável dependente, tipo respiratório e modo respiratório. 0 nível de significância foi de $5 \%$ ( $p \leq$ $0,05)$.

\section{Resultados}

Os resultados obtidos são apresentados nas tabelas de um a cinco, expostas a seguir.

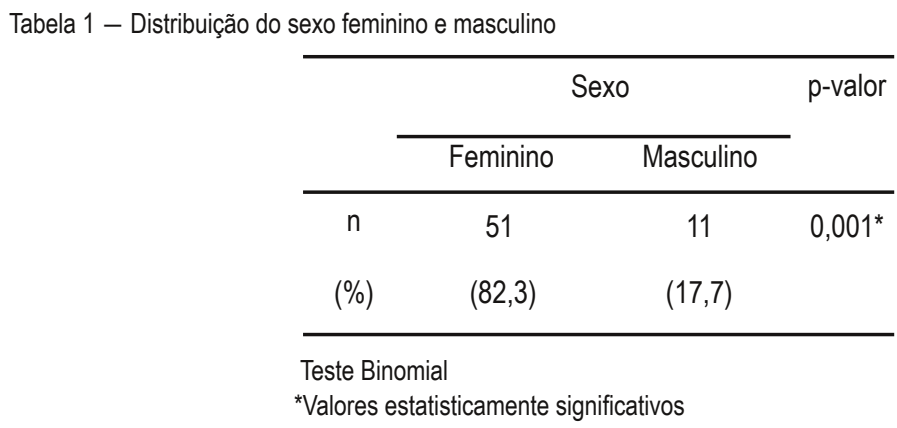

Saúde (Santa Maria), v.39, n.1, p. 121-130, 
Tabela 2 - Distribuição dos tipos respiratórios em repouso e durante a fala entre os sexos

\begin{tabular}{cccccc}
\hline \multicolumn{5}{c}{ Tipo respiratório em repouso } \\
\hline Sexo & CDA & Misto & Superior & Torácico & p-valor \\
\hline Feminino & 7 & 43 & 1 & 0 & 0,083 \\
Masculino & 2 & 7 & 1 & 1 & \\
$\mathrm{n}(\%)$ & $(3,2)$ & $(11,29)$ & $(1,6)$ & $(1,6)$ & \\
\hline Feminino & 7 & 43 & 1 & 0 & 0,095 \\
$\mathrm{n}(\%)$ & $(11,2)$ & $(69,3)$ & $(1,6)$ & $(0)$ & \\
Masculino & 1 & 8 & 1 & 1 & \\
$\mathrm{n}(\%)$ & $(1,6)$ & $(12,9)$ & $(1,6)$ & $(1,6)$ & \\
\hline
\end{tabular}

Teste qui-quadrado

Legenda: $\mathrm{CDA}=$ costodiafragmáticoabdominal

Tabela 3 - Distribuição dos modos respiratórios em repouso e durante a fala entre os sexos

\begin{tabular}{ccccc}
\hline \multicolumn{5}{c}{ Modo respiratório em repouso } \\
\hline Sexo & Nasal & Oral & Oronasal & p-valor \\
\hline Feminino & 47 & 2 & 3 & 0,095 \\
Masculino & 9 & $(3,2)$ & $(4,8)$ & \\
n (\%) & $(75,8)$ & 0 & 2 & \\
\hline Feminino & 1 & $(0)$ & $(3,2)$ & 0,707 \\
n (\%) & $(1,6)$ & $(3,5)$ & $(75,8)$ & \\
Masculino & 0 & 0 & 11 & \\
\hline Teste qui-quadrado & & $(0)$ & $(17,7)$ & \\
\hline
\end{tabular}

Teste qui-quadrado

Tabela 4 - Comparação entre as proporções da variável tipo respiratório em repouso e durante a fala

\begin{tabular}{ccc}
\hline Tipo respiratório em repouso & p-valor \\
\hline CDA & Misto & $0,002^{*}$ \\
$n(\%)$ & $50(80,6)$ & \\
$9(14,5)$ & Superior & \\
CDA &
\end{tabular}

$9(14,5) \quad 2(3,2)$

Rev. Saúde (Santa Maria), v.39, n.1, p. 121

130, Jan./Jul. 2013.

Hoffmann, C. et al. 


\begin{tabular}{|c|c|c|}
\hline $\mathrm{CDA}$ & Torácico & 0,617 \\
\hline $9(14,5)$ & $1(1,6)$ & \\
\hline Misto & Superior & $0,010^{*}$ \\
\hline $50(80,6)$ & $2(3,2)$ & \\
\hline Misto & Torácico & $0,010^{*}$ \\
\hline $50(80,6)$ & $1(1,6)$ & \\
\hline Superior & Torácico & 0,948 \\
\hline $2(3,2)$ & $1(1,6)$ & \\
\hline \multicolumn{2}{|c|}{ Tipo respiratório durante a fala } & p-valor \\
\hline CDA & Misto & $0,001^{*}$ \\
\hline$n(\%)$ & $n(\%)$ & \\
\hline $8(12,9)$ & $51(82,2)$ & \\
\hline CDA & Superior & 0,702 \\
\hline $8(12,9)$ & $2(3,2)$ & \\
\hline CDA & Torácico & 0,625 \\
\hline $8(12,9)$ & $1(1,6)$ & \\
\hline Misto & Superior & $0,009^{*}$ \\
\hline $51(82,2)$ & $2(3,2)$ & \\
\hline Misto & Torácico & $0,008^{*}$ \\
\hline $51(82,2)$ & $1(1,6)$ & \\
\hline Superior & Torácico & 0,946 \\
\hline $2(3,2)$ & $1(1,6)$ & \\
\hline
\end{tabular}

Teste Binomial

*valores estatisticamente significativos

Legenda: $\mathrm{CDA}=$ costodiafragmáticoabdominal

Tabela 5 - Comparação entre as proporções da variável modo respiratório em repouso e durante a fala

\begin{tabular}{ccc}
\hline \multicolumn{3}{c}{ Modo respiratório em repouso } \\
\hline Nasal & Oral & p-valor \\
$56(90,3)$ & $n(\%)$ & $0,005^{*}$ \\
Nasal & $1(1,6)$ & \\
$56(90,3)$ & Oronassal & $0,001^{*}$ \\
Oral & $5(8,1)$ & \\
$1(1,6)$ & Oronasal & 0,354 \\
\hline
\end{tabular}




\begin{tabular}{ccc}
\hline \multicolumn{3}{c}{ Modo respiratório durante a fala } \\
\hline Nasal & Oral & p-valor \\
$n(\%)$ & $n(\%)$ & \\
$1(1,6)$ & $1(1,6)$ & 0 \\
Nasal & Oronassal & \\
$1(1,6)$ & $58(93,5)$ & $0,001^{*}$ \\
Oral & Oronasal & \\
$1(1,6)$ & $58(93,5)$ & $0,001^{*}$ \\
\hline
\end{tabular}

Teste Binomial

*valores estatisticamente significativos

\section{Discussão}

Predominaram participantes do sexo feminino (Tabela 1), provavelmente pela maior procura deste gênero por atendimentos de saúde ${ }^{15}$. Como não houve diferença significativa comparando-se o tipo e o modo respiratório em relação aos sexos (Tabelas 2 e 3), optou-se por realizar a análise das variáveis estudadas no grupo como um todo (Tabelas 4 e 5).

A respiração nasal é importante para o bom funcionamento da produção vocal, sendo de fundamental importância quando se trata de indivíduos que fazem uso da voz profissionalmente $e^{5-7,30}$. 0 modo respiratório incorreto pode influenciar 0 estabelecimento e 0 prognóstico dos distúrbios vocais, muitas vezes negligenciadas em detrimento de outros distúrbios como os de fala, mastigação e deglutição causados pela respiração oral|5,7,9,23.

Os distúrbios vocais provocados pelo modo respiratório incorreto englobam desvios no sistema de ressonância e na projeção vocal, o que provoca esforço laríngeo como mecanismo compensatório que, por sua vez, pode levar ao surgimento de afecções laríngeas ${ }^{5,7,23}$. Em uma pesquisa, foi observada relação significativa de sujeitos com disfonia organofuncional e queixa de distúrbios alérgicos, causadores de respiração oral ${ }^{23}$.

O grau de influência de fatores como a respiração oral sobre a voz não é linear, variando entre os indivíduos ${ }^{23}$. De forma geral, a respiração oral, causa pobre ventilação das fossas nasais, aumentando as secreções que podem banhar a laringe, o que causa irritação e edema, além da sensação de corpo estranho e pigarro, aumentando o atrito durante a vibração das pregas vocais ${ }^{23,24}$. A respiração oral também causa ressecamento laríngeo, bem como choque térmico e acúmulo de partículas do ar sobre 0 epitélio das pregas vocais, devido à perda das funções de umidificação, aquecimento e filtragem realizadas pelo nariz ${ }^{24}$.

Em casos alérgicos, o tratamento também pode gerar consequências na produção vocal, pois os medicamentos usados possuem efeito ressecante no trato vocal, podendo gerar sobreesforço e maior atrito da mucosa das pregas vocais durante a fonação?2.

No presente estudo, a maioria significativa dos sujeitos apresentou respiração nasal durante o repouso e oronasal durante a fala (Tabela 5), consideradas ideais para a saúde vocal. 0 mesmo resultado foi encontrado em outras duas pesquisas, uma com 23

Rev. Saúde (Santa Maria), v.39, n.1, p. 121 130, Jan./Jul. 2013 Hoffmann, C. et al. 
profissionais da voz guias de turismo e outra com 26 coralistas, cuja maioria dos sujeitos apresentou modo respiratório nasal durante o repouso ${ }^{10,11}$.

O tipo respiratório inadequado, quando associado à intensa demanda vocal também pode contribuir negativamente com a produção da voz, pois uma respiração que não utiliza todo o suporte aéreo necessário, geralmente implica em sobre-esforço vocal2,6,8,16,28.

Dos quatro diferentes tipos respiratórios, o superior é considerado prejudicial para o uso profissional da voz, pois ocorre elevação das clavículas na inspiração, com consequente aumento de tensão de toda a região cervical e pouca movimentação do abdômen ${ }^{5,28}$. No tipo respiratório inferior, não ocorre movimentação da região superior do tórax, sendo o abdômen o responsável pela maior parte do movimento, não havendo aproveitamento de todo suporte respiratório de que a voz profissional necessita, além de favorecer uma inadequada postura corporal, causadora de hipertensões musculares 2,10,16,30.

O tipo misto, em que ocorre pouca elevação da região superior e inferior do tórax, é aceito na fala cotidiana e no repouso. Porém é contraindicado durante o uso profissional da voz devido ao suporte aéreo insuficiente, podendo ocorrer cansaço para falar, instabilidade vocal, falta de ar ao final das frases, dentre outras queixas que, associadas à grande demanda vocal, poderão desencadear distúrbios vocais ocupacionais 2,10,16,30.

O tipo CDA caracteriza-se pela expansão harmônica da caixa torácica, sem excessos na região superior. Proporciona maior equilíbrio na emissão do ar durante o discurso, determinando uma fonação mais adequada, sem esforço. Através do tipo CDA, a coluna se mantém ereta e, como consequência, os músculos abdominais se contraem. Juntamente com o músculo diafragma, os abdominais promovem maior aproveitamento do ar expiratório e maior controle da pressão e do fluxo aéreo, importantes tanto no uso da emissão vocal falada quanto cantada ${ }^{2,10,16,30}$.

No presente estudo, a maioria significativa apresentou tipo respiratório misto em repouso e durante a fala (Tabela 4), o que é um aspecto preocupante sobre a saúde vocal desses futuros profissionais da voz. 0 tipo respiratório inadequado durante o uso profissional da voz pode ser responsável pelo estabelecimento de disfonias funcionais e posteriormente organofuncionais, agregando ao quadro os sintomas vocais desconfortáveis e o desempenho profissional prejudicado. Tal achado concorda com a literatura que mostra que o tipo respiratório misto predomina em profissionais da voz, associado a outras alterações da produção vocal ${ }^{10,11}$.

Referente ao preparo necessário para uma intensa demanda vocal, os professores de música realizam exercícios com foco na contração da musculatura abdominal e no apoio respiratório $8,16,29,30$. Em pesquisa com instrumentistas de sopro, concluiu-se que 0 tipo respiratório mais presente foi o CDA pelo fato dos sujeitos necessitarem de respiração mais completa durante a execução dos movimentos de sopro ${ }^{30}$.

A prática do tipo respiratório CDA deve ser difundida aos profissionais da voz em geral. Através do treinamento respiratório adequado e associado a exercícios vocais específicos, a produção vocal poderá acompanhar as necessidades vocais profissionais de maneira natural. O conhecimento sobre o funcionamento do sistema respiratório, assim como seu domínio

Saúde (Santa Maria), v.39, n.1, p. 121-130,

2013. $\mathrm{voz}^{3,4,31}$ 


\section{Conclusão}

A maioria dos futuros profissionais da voz, de ambos os sexos, deste estudo apresentou modo respiratório adequado durante a fala e o repouso. No entanto, o tipo respiratório mais frequente foi o misto, tanto durante a fala quanto em repouso, sendo considerado prejudicial para a demanda de voz profissional.

Desta forma, verifica-se a necessidade de intervenção fonoaudiológica, a fim de orientar sobre cuidados vocais e treinar o tipo respiratório correto durante o uso da voz profissional. Tal ação assume um caráter preventivo quando realizada com futuros profissionais da voz, para evitar e/ou diminuir os gastos públicos com afastamentos e tratamentos devido a distúrbios vocais.

\section{Referências Bibliográficas}

1. Rossi DC, Munhoz DF, Nogueira CR, Oliveira TCM, Britto ATBO. Relação do pico de fluxo expiratório com o tempo de fonação em pacientes asmáticos. Rev CEFAC. 2006;8(4)509-17.

2. Behlau M. Voz: O livro do Especialista. Rio de Janeiro: Revinter, 2008.

3. Ueda KH, Santos LZ, Oliveira IB. 25 Anos de cuidados com a voz profissional: avaliando ações. Rev CEFAC. 2008;10(4):557-65.

4. Fabrício MZ, Kasama ST, Martinez EZ. Qualidade de vida relacionada à voz de professores universitários. Rev CEFAC. 2009;12(8):183-08.

5. Tavares JG, Silva VA, Alves EHA. Considerações teóricas sobre a relação entre respiração oral e disfonia. Rev Soc Bras Fonoaudiol. 2008;13(4):405-10.

6. Carneiro PR. Características acústicas da voz em diferentes posturas corporais [dissertação]. São Carlos (SP): Universidade de São Paulo. Programa de Pós-Graduação Interunidades em Bioengenharia/ EESC/ FMRP/ IQSC; 2009.

7. Nishimura CM, Gimenez SRML. Perfil da fala do respirador oral. Rev CEFAC. 2009;12(3):505-08.

8. Barbiero EF, Vanderlei LCM, Nascimento PC, Costa MM, Scalabrini Neto A. Influência do biofeedback respiratório associado ao padrão quiet breathing sobre a função pulmonar e hábitos de respiradores bucais funcionais. Rev Bras Fisioter. 2007;11(5):347-53.

9. Bianchini AP, Guedes ZFC, Vieira MM. Estudo da relação entre respiração oral e tipo facial. Rev Bras Otorrinolaringol. 2007; 73(4):500-05.

10. Soares EB, Brito CMCP. Hábito e perfil vocal em coralistas. Acta ORL. 2009;27(1):28-35.

11. Soares EB, Brito CMCP. Perfil vocal do guia de turismo. Rev CEFAC. 2006;8(4):501-08.

12. Santana MCCP, Brandão KKCP, Goulart BNG, Chiari BM. Fonoaudiologia e saúde do trabalhador: vigilância é informação para a ação! Rev CEFAC. 2009;11(3)522-28.

13. Silvério KCA, Pereira EC, Menoncin LM, Dias CAS, Santos CLG, Schwartzman PP. Avaliação vocal e cervicoescapular em militares instrumentistas de sopro. Rev Soc Bras Fonoaudiol. 2010;15(4):497-504.

14. Lierde KM, Claeys S, Dhaeseleer E, Deley S, Derde K, Herregods I, Strybol I, Wuyts F. Vocal quality in female student teachers. J Voice. 2010;24(5):599-605.

Rev. Saúde (Santa Maria), v.39, n.1, p. 121

130, Jan./Jul. 2013.

15. Christmann MK, Scherer TM, Hoffmann CF, Cielo CA. Tempo máximo de fonação de futuros profissionais da voz. Rev CEFAC [prelo]. 
16. Andrade SR, Fontoura DR, Cielo CA. Interrelações entre fonoaudiologia e canto. Rev Música Hodie. 2007;7(1):83-98.

17. Andrews ML. Manual de tratamento da voz: da pediatria à geriatria. São Paulo: Cengage Learning, 2009.

18. Kurtz LO, Cielo CA. Tempos máximos de fonação de vogais em mulheres adultas com nódulos vocais. Pró-fono. 2010;22(4):451-54.

19. Cielo CA, Lasch SS, Miglioranzi SL, Conterno G. Tempos máximos de fonação e características vocais acústicas de mulheres com nódulos vocais. Rev CEFAC. 2011; 13(3):437-43.

20. Gelfer MP, Pazera JF. Maximum duration of sustained $/ \mathrm{s} /$ and $/ z /$ and the $s / z$ ratio with controlled intensity. J Voice. 2006;20(3):369-79.

21. Quedas A, Duprat AC, Gasparini G. Implicações do efeito Lombard sobre a intensidade, frequência fundamental e estabilidade da voz de indivíduos com doença de Parkinson. Rev Bras Otorrinolaringol. 2007;73(5):675-83.

22. Cielo CA, Cappellari VM. Tempo máximo de fonação de crianças pré-escolares. Rev Bras Otorrino. 2008;74(4):552-60.

23. Cielo CA, Finger LS, Roman-Niehues G, Deuschle VP, Siqueira MA. Disfonia organofuncional e queixas de distúrbios alérgicos e/ou digestivos. Rev CEFAC. 2009;11(3):431-39.

24. Pinho SMR. Manual de higiene vocal para profissionais da voz. Carapicuíba: Pró-Fono, 2007.

25. Farghaly SM, Andrade CRF. Programa de treinamento vocal para locutores de rádio. Rev Soc Bras Fonoaudiol. 2008;13(4):316-24.

26. Pinho SMR, Pontes P. Escala de avaliação perceptiva da fonte glótica: RASATI. Vox Brasilis. 2002; 3(1):11-3.

27. Costa JO, Gama ACC, Oliveira JBO, Neto ALR. Avaliação acústica e perceptivo-auditiva da voz nos momentos pré e pós-operatório da cirurgia de implante de pré-fáscia do músculo temporal. Rev CEFAC. 2008;10(1):76-83.

28. Silvério KCA, Pereira EC, Menoncin LM, Dias CAS, Santos Junior CLG, Schwartzman PP. Avaliação de instrumentistas de sopro. Rev Soc Bras Fonoaudiol. 2010;15(4):497-504.

29. Crispiniano T, Bommarito S. Avaliação da musculatura orofacial e postura corporal em pacientes com respiração bucal e maloclusão. Rev Odonto. 2007;15(29):88-97.

30. Gava Júnior W, Ferreira LP, Andrada e Silva MA. Apoio respiratório na voz cantada: perspectiva de professores de canto e fonoaudiólogos. Rev CEFAC. 2010;12(4):551-52.

31. Araújo MT, Reis EJFB, Carvalho M., Porto LA, Reis IC, Andrade JM. Fatores associados a alterações vocais em professoras. Cad Saúde Publ. 2008;24(6): 1229-38.

\section{Carla Aparecida Cielo}

Endereço para correspondência - Rua Guilherme João Fabrin, 545. CEP: 97050-280, Santa Maria, RS, Brasil.

E-mail: cieloca@yahoo.com.br

Currículo Lattes: http://lattes.cnpq.br/8424979142095675

Saúde (Santa Maria), v.39, n.1, p. 121-130,
Recebido em 25 de outubro de 2012

Aprovado em 23 de julho de 2013 